\title{
Dynamic noise modeling at roundabouts
}

\author{
E. Chevallier ${ }^{\mathrm{a}}$, L. Leclercq ${ }^{\mathrm{a}, *}$, J. Lelong ${ }^{\mathrm{b}}$, R. Chatagnon ${ }^{\mathrm{b}}$ \\ a Université de Lyon, ENTPE/INRETS - Laboratoire d'Ingénierie Circulation Transport, Rue Maurice Audin, 69518 Vaulx-en-Velin Cedex, France \\ ${ }^{\mathrm{b}}$ INRETS - Laboratoire Transport Environnement, 25 Avemue François Mitterrand, 69675 Bron Cedex, France
}

\section{A R T I C L E I N F O}

\section{Article history:}

Received 19 March 2008

Received in revised form 5 September 2008

Accepted 12 September 2008

Available online 5 November 2008

\section{Keywords:}

Urban traffic noise

Roundabout

Dynamic

Noise mapping

Interrupted traffic

Traffic simulation

\begin{abstract}
A B S T R A C T
Modeling spatial and temporal noise variations at roundabouts is a tedious task. Indeed, noise levels are strongly influenced by the complex vehicle interactions taking place at the entries. An accurate modeling of the merging process and its impact on vehicle kinematics, waiting time at the yield signs and queue length dynamics is therefore required. Analytical noise prediction models disregard those impacts since they are based on average flow demand patterns and pre-defined kinematic profiles. The only way to capture all traffic dynamics impacts on noise levels is to combine a traffic simulation tool with noise emission laws and a sound propagation model. Yet, such existing dynamic noise prediction packages fail in representing vehicle interactions when the roundabout is congested and are difficult to calibrate due to their numerous parameters. A new traffic simulation tool, specifically developed for roundabouts, is therefore proposed in this paper. It has few easy-to-calibrate parameters and can be readily combined with noise emission and propagation laws. The obtained noise package is able to produce relevant dynamic noise contour maps which can support noise emission assessment of local traffic management policies. Results are validated against empirical data collected on a French suburban roundabout on two different peak periods.
\end{abstract}

(c) 2008 Elsevier Ltd. All rights reserved.

\section{Introduction}

Space-time variations in vehicle kinematics due to intersections were shown to greatly influence noise levels in urban areas $[1,2]$. Although several noise estimation procedures have been developed to catch these effects, few of them are suitable for improving noise mapping at roundabouts.

In most existing prediction tools, like the FHWA Traffic Noise Model [3] or the German RLS-90 Model [4], noise impacts of interrupted traffic flows are roughly taken into account through empirical correction factors. More sophisticated statistical models have been proposed to improve noise appraisal at signalized intersections in terms of traffic volumes, traffic speeds, traffic composition, geometry or pavement surface textures [5]. Derivation of similar regression formula at roundabouts is possible, yet unfruitful since spatial variations in noise levels cannot be captured.

To circumvent this deficiency, advanced analytical methodologies based on the computation of average noise signatures for each vehicle class have been introduced [6,7]. They combine a mean vehicle kinematic pattern per class with a noise emission law depending on vehicle speed and running conditions. A single class-specific vehicle is assumed to cross the junction. At each instant, the resulting sound power level and vehicle position are input into a propagation model to calculate the instantaneous sound

\footnotetext{
* Corresponding author. Tel.: +33 472047716; fax: +33 472047712 .

E-mail address: leclercq@entpe.fr (L. Leclercq).
}

pressure level at a given receptor point. The sound pressure exposure of the class-specific vehicle is then obtained by integrating the instantaneous sound pressure levels over the time needed to cross the junction. Repeating this process for a set of receivers along the infrastructure gives the average noise signature for the corresponding vehicle class. Influence of traffic flow can then be accounted for by multiplying the noise signature by the number of vehicles passing during a given interval and so, for each class. Such a method has already been applied to roundabouts [8]. It has also been implemented in the Harmonoise traffic source model to draw noise difference contour maps at road crossings between situations where all vehicles maintain constant speed without stopping and situations where they all have to decelerate, stop and then accelerate away from the junction [9]. Spatial kinematic variations appeared to either increase or decrease average noise levels depending on the traffic volumes. The major drawback of this analytical procedure is to neglect the noise impacts of vehicle interactions. Particularly, it cannot catch noise variations due to: (i) random occurrence of stopping or freely-entering vehicles into the roundabout; (ii) fluctuating waiting times on the roundabout approaching links; (iii) fluctuating queue lengths; and (iv) potential hindrance effect due to a congestion spilling back from downstream. As a result, the output noise contour maps are not completely satisfactory.

The only way to catch the noise impacts of vehicle interaction consists in coupling a traffic flow simulation tool with vehicle noise emission laws and a sound propagation model. As underlined 
within the Imagine project [10] this technique allows for evaluating how short-term transient queue events as well as spatial and temporal variations in vehicle kinematics influence noise estimates. It has already been implemented into softwares like DRONE [11], MOBILEE [12], TUNE [13] or ROTRANOMO [14]. However, to the authors' knowledge, no model calibration or validation study was specifically conducted to assess noise estimates at roundabouts. This is troublesome since the corresponding traffic simulation tools included into these softwares (respectively AVENUE or $M+P$, PARAMICS, DRACULA, VISSIM) have a large number of parameters that are often difficult to calibrate and may affect noise outputs to a large extent. Moreover, these tools were recently shown to badly represent vehicle interactions and kinematics in highly congested situations [15,16]. By predicting unrealistic queue length and delay values, they may, therefore, lead to irrelevant noise estimates at roundabouts.

The goal of this paper is to propose another dynamic noise emission model based on a new microscopic traffic simulation tool, specifically devoted to roundabouts. This tool solves the major drawbacks of classical micro-simulation packages underlined within the IMAGINE project: (i) it has few parameters; (ii) it is easy-to-calibrate with a limited set of data; (iii) it is able to model the number of stopped vehicles, the vehicle delays and the queue length dynamics on each approaching links accurately whatever the traffic conditions on the roundabout. Consequently, realistic vehicle kinematics can be obtained and fed into appropriate noise emission laws. Then, a sound propagation calculation is performed with respect to geometry and urban landscape. This paper will highlight that accurate dynamic noise contour maps can be simulated. They can be used to assess the noise impacts of traffic flow at roundabouts in both free-flow and saturated conditions.

The first part of this paper will outline the three key components of the proposed framework: the microscopic traffic simulation tool, the noise emission laws and the sound propagation model. For the study purpose, we will only focus on: (i) a single mean emission law for light duty vehicles; (ii) a very basic propagation model. However, it should be highlighted that the framework can handle with any class-specific emission laws and very complex propagation models. In the second part of the paper, the overall framework will be validated against empirical sound pressure levels collected at four receptor points close to the road axis of a single-lane roundabout. Results in terms of equivalent sound pressure levels, statistical descriptors as well as noise levels distributions are really convincing.

\section{Basic components of the roundabout noise emission model}

\subsection{Overview of the model}

The purpose of the roundabout noise emission model is to accurately account for traffic dynamics in order to reproduce spatial and short-term variations in noise levels in the vicinity of the infrastructure. As mentioned in the introduction, this can be done by coupling a traffic flow simulation tool with noise emission laws and a sound propagation model. The modeling chain is depicted in Fig. 1. The traffic flow simulation needs some input data to fix the traffic demand scenario (traffic volumes, destination proportions, traffic composition) as well as some parameters which has to be calibrated beforehand. Outputs of the traffic model are position $x^{t}$, speed $\vartheta$, acceleration $a^{t}$ and vehicle type of each vehicle, at each time $t$. Those outputs are fed into noise emission laws to assign an instantaneous sound power level $L_{w}^{t}$ to each vehicle. Then, individual contributions of each vehicle at a given receptor point can be calculated according to a sound propagation model. The instantaneous sound pressure levels at the receiver are obtained by summing all the contributions at each time. Finally, those levels can be used to calculate a large array of noise descriptors.

\subsection{Microscopic traffic simulation tool}

Microscopic traffic simulation tools aim at modeling the progression of individual vehicles through a road network. The overall simulation period is usually broken down into a number of discrete time-steps $\Delta t$. Positions of all vehicles are updated at each timestep by specific algorithms. Speed and acceleration can then be deduced from positions at successive time-steps. Two algorithms are commonly used in order to model a roundabout: (i) a car-following algorithm which simulates vehicle trajectories on the approaching/ departure links and inside the roundabout (the position of any vehicle in the network is given in terms of the one of its leader); (ii) an insertion decision algorithm which specifies whether vehicles arriving at the yield sign can enter or not the roundabout.

Most of the existing microscopic simulation tools for roundabouts were shown to have the following drawbacks:

- profusion of parameters that may be troublesome or data consuming to calibrate [17];

- sensitivity of the simulated vehicle trajectories to parameters [18];

- failure in modeling insertion rates, vehicle delays and queue length dynamics when a congestion spills back on the roundabout $[15,16]$.

As a result, reliability of noise level estimates cannot be guaranteed when these traffic flow models are used in combination with a noise estimation process.

This has motivated the development of a new parsimonious, easy-to-calibrate microscopic traffic model for roundabouts able to capture two kinds of observed merging behaviours depending on traffic conditions on the roundabout:

- in free-flow conditions, approaching vehicles respect the yield rule and insert into the roundabout only if the time-interval before the arrival of the next circulating vehicle is sufficient [19];

- in congested conditions, approaching vehicles do not respect the yield rule anymore and alternatively insert between circulating vehicles following a ratio $\gamma[20,21]$.

To model these merging behaviours, two distinct insertion decision algorithms are used:

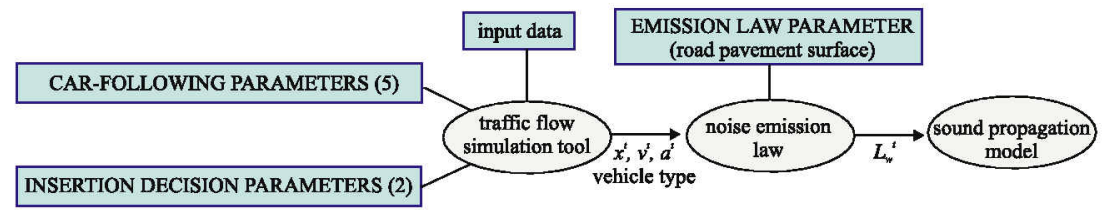

$\subseteq$ components of the noise simulation model $\square$ parameters or input data (need mesurements)

Fig. 1. Overview of the dynamic roundabout noise emission model. 


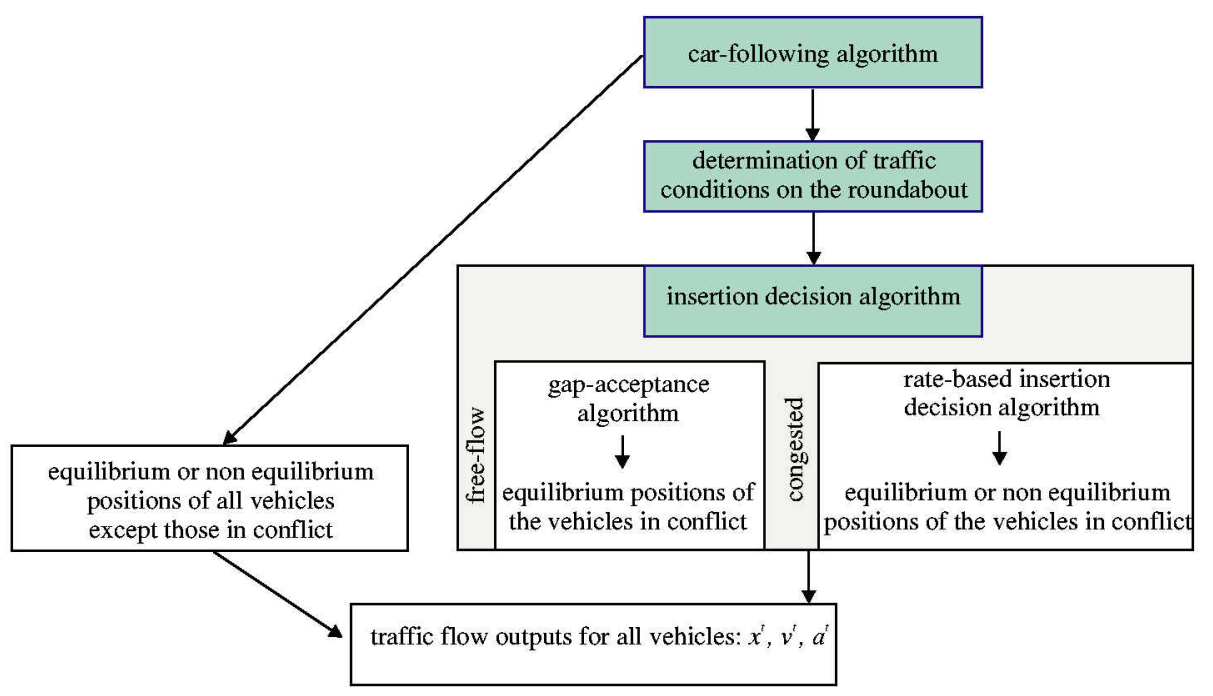

Fig. 2. Flowchart of the microscopic traffic simulation model.

- a gap-acceptance algorithm in free-flow conditions which specifies minimum distance and time-interval criteria to allow for an insertion;

- a rate-based algorithm in congested conditions which specifies the probability for an approaching vehicle to enter into the roundabout.

Fig. 2 summarizes the main steps of the microscopic simulation model. Firstly, positions of all vehicles except those in conflict (the approaching vehicle who wants to enter the roundabout and the next circulating vehicle on the circulatory roadway who is going to arrive at the conflict point) are updated according to the car-following model. Then, traffic conditions on the roundabout are assessed before implementing the appropriate insertion decision algorithm. Those model components will be described in the sequel; more details can be found in Chevallier and Leclercq [16].

\subsubsection{Car-following algorithm}

In equilibrium traffic conditions on the roundabout, the velocity of vehicle $n$ at time $t, v_{n}^{t}$, only depends on the spacing $s_{n}^{t}$ with the vehicle ahead $n-1$. The relationship $v_{n}^{t}=v_{e}\left(s_{n}^{t}\right)$ is classically referred to as the fundamental diagram. It is depicted in Fig. 3. It requires only three parameters: (i) the speed in free-flow conditions $u$; (ii) the minimum spacing when vehicles are stopped $s_{0}$; (iii) the speed at which a queue spills back on the network $w$. Free-flow conditions correspond to points on the fundamental diagram where both spacing and velocity are high while congested conditions match the other part of the curve.

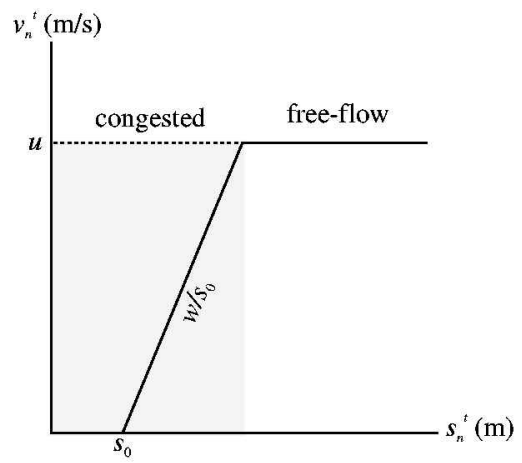

Fig. 3. Fundamental diagram.
Because of aggressive insertions from the approaching links, $s_{n}^{t}$ may shorten below its equilibrium value. In this case, experimental studies have demonstrated that vehicle $n$ adapts its speed to gradually increase the spacing with its leader. In other words, it tolerates short spacing for a while without braking sharply. This process is called a relaxation procedure. In the remaining, we denote $\beta_{n}^{t}$ the ratio between the current spacing in front of vehicle $n$ and the equilibrium spacing (given by the fundamental diagram with respect to the speed of the leader). The relaxation process of vehicle $n$ starts as soon as another vehicle inserts just ahead with a very short spacing and lasts until $\beta_{n}^{t}=1$. In the chosen car-following model, the time evolution of $\beta_{n}^{t}$ only depends on one additional parameter, the relaxation parameter, $\epsilon$, corresponding to the difference in speed drivers are willing to accept with the vehicle ahead in order to recover an equilibrium state (see Laval and Leclercq [22] for more details). For instance, when two vehicles are closely spaced on the roundabout, $\epsilon$ is approximately the difference between the speed of the leader at the previous time-step and the desired speed of the follower for the current time-step.

Eventually, the position of vehicle $n$ at time $t+\Delta t, x_{n}^{t+\Delta t}$, is given as the minimum between the position it is willing to reach when traffic is in free-flow condition and the position it cannot overpass due to the downstream vehicle $n-1$ when traffic is congested:

$$
\begin{aligned}
x_{n}^{t+\Delta t}= & \min [\underbrace{x_{n}^{t}+\min \left(u, v_{n}^{t}+a \Delta t\right) \Delta t}_{\text {position reachable in free-flow }} ; \\
& \times \underbrace{(1-\alpha) x_{n}^{t}+\alpha x_{n-1}^{t}+v_{n-1}^{t} \Delta t-\alpha \beta_{n}^{t+\Delta t} s_{e}\left(v_{n-1}^{t+\Delta t}\right)}_{\text {position imposed by the leader in congestion }}]
\end{aligned}
$$

In Eq. (1), $a$ is the desired acceleration common to all vehicles; $s_{e}$ is the reciprocal of the fundamental diagram which gives the equilibrium spacing in front of a vehicle when its velocity is known; $\alpha=\min \left[1 ; w \Delta t /\left(s_{0} \beta_{n}^{t}\right)\right]$ is a non-dimensional coefficient. Eq. (1) is used to model vehicle trajectories on the approaching and departure arms as well as inside the roundabout. To account for different driver behaviours inside the roundabout, the parameters of the fundamental diagram can be modified (for instance, the speed in freeflow conditions can be reduced).

\subsubsection{Insertion decision algorithms}

According to the current spacing of the first downstream circulating vehicle and the fundamental diagram, the prevailing traffic 
conditions on the roundabout (free-flow or congested) can be specified to choose the appropriate insertion decision algorithm.

Free-flow state: The gap-acceptance algorithm allows for an insertion into the roundabout during the current time-step if:

- A time $t_{f}$ has elapsed since the last circulating vehicle has passed by the entry. This criteria is equivalent to assuming a maximum inserting flow of $1 / t_{f}$ which usually depends on the roundabout radius, the road gradient, or visibility constraints.

- The next circulating vehicle expected to pass by the entry is beyond a distance $d_{\text {lag }}$ which is sufficient to avoid collision in case of an insertion. This distance is given as the product of the free-flow speed $u$ and the difference between the minimum time-headway between two circulating vehicles that an approaching driver consider to be sufficient to enter the roundabout, $t_{c}$ (which is a model parameter), and $t_{f}$.

- The inserting vehicle has enough space to enter the roundabout, that is to say the position imposed by the next vehicle (second term of the minimum function in Eq. (1)) does not lead the inserting vehicle to move backwards.

These conditions can be assessed thanks to the positions of all vehicles except those in conflict given by the car-following algorithm. In case of insertion, the positions of the two vehicles in conflict are updated according to the car-following model (Eq. (1)) operating in equilibrium conditions $\left(\beta_{n}^{t}=1\right)$ since no aggressive insertion are assumed to occur in free-flow state.

Congested state: The rate-based insertion decision model aims at simulating an average insertion flow equal to $\gamma$ times the circulating flow on the roundabout passing by the entry. For this, on each approaching $\operatorname{arm} i$, the average traffic volumes just upstream of the entry, $\Delta_{i}$, and just downstream of the entry on the roundabout, $\Omega_{i}$, should be computed (over the last $30 \mathrm{~s}$ for instance). Then, as soon as an approaching vehicle is close enough to the yield-sign, the probability for it to enter the roundabout during the time-step, $p^{t+\Delta t}$, is computed from Eq. (2) (see Chevallier and Leclercq [16]):

$p^{t+\Delta t}= \begin{cases}\Omega \gamma /(1+\gamma) \Delta t & \text { if } \Delta_{i} \geqslant \Omega \gamma /(1+\gamma) \\ 1 & \text { otherwise }\end{cases}$

A Bernoulli process is then drawn to specify the outcome of the insertion process. In case of insertion, both vehicles in conflict may switch to a non-equilibrium state $\left(\beta_{n}^{t}<1\right)$ or stay in equilibrium $\left(\beta_{n}^{t}=1\right)$ depending on the position of their leader. Their new positions are then calculated according to the car-following Eq. (1).

\subsubsection{Input data}

As any micro-simulation tool, the traffic flow model requires input data to specify the prevailing traffic demand scenario. Data can either be precisely measured or scaled from aggregated traffic observations (see Table 1).

Table 1

Input data depending on the level of detail of on-site measurements

\begin{tabular}{|c|c|c|}
\hline Input data & Precise on-site measurements & Aggregated traffic observations \\
\hline $\begin{array}{l}\text { Traffic } \\
\quad \text { volume }\end{array}$ & $\begin{array}{l}\text { Arrival times of all vehicles at } \\
\text { the beginning of each } \\
\text { approaching arm }\end{array}$ & $\begin{array}{l}\text { Time-series of average flows at } \\
\text { the beginning of each } \\
\text { approaching arm }\end{array}$ \\
\hline Destination & $\begin{array}{l}\text { Destination of each vehicle } \\
\text { entering the approaching arms }\end{array}$ & $\begin{array}{l}\text { Turning proportions for each } \\
\text { approaching stream }\end{array}$ \\
\hline Traffic & $\begin{array}{l}\text { Type of each vehicle entering the } \\
\text { approaching arms }\end{array}$ & $\begin{array}{l}\text { Proportion of each vehicle class } \\
\text { within the flow }\end{array}$ \\
\hline $\begin{array}{r}\text { Vehicle } \\
\text { type }\end{array}$ & $\begin{array}{l}\text { proportions for each } \\
\text { approaching stream }\end{array}$ & \\
\hline
\end{tabular}

\subsection{Noise emission laws}

The traffic model outputs are fed into noise emission laws in order to calculate the instantaneous sound power levels, $L_{w n}^{t}$, for any vehicle $n$ on the roundabout. Any noise emission law in terms of vehicle type, vehicle speed and/or acceleration can be used. In this study, a single mean emission law is selected to characterize all vehicles on the roundabout (mainly light-duty vehicles as we will see later). This hypothesis was validated in Can et al. [23] for classical descriptors estimation in urban area, provided that traffic dynamics is precisely described. The chosen emission law is the average law for light-duty vehicles used within the French traffic noise prediction model [24] and corresponding to the pavement surface texture of the studied experimental site (see Section 3 ). It has two main advantages: (i) it is specifically devoted for urban traffic conditions; (ii) it has been validated in real life traffic conditions, for instance when vehicles are accelerating away from a signalized intersection (low speeds and high accelerations) at different points of a urban corridor [25].

This emission law, depicted in Fig. 4a, depends on vehicle speed and running conditions which are specified by the vehicle acceleration: (i) accelerating mode for $a>0.5 \mathrm{~ms}^{-2}$; (ii) decelerating mode for $a<0.5 \mathrm{~ms}^{-2}$; (iii) cruising mode otherwise. For speeds below $30 \mathrm{~km} / \mathrm{h}$, the engine-exhause noise is predominant [10]. The value of $L_{w n}^{t}$ depends on the running conditions of $n$ : in cruising or deceleration modes, it follows a logarithmic function truncated for low speeds; in acceleration mode it reaches a higher constant level. For speeds above $30 \mathrm{~km} / \mathrm{h}$, the tire-pavement noise prevails upon the engine-exhause noise: no distinction in running conditions is made for the calculation of $L_{w n}^{t}$ since, in urban areas, the acceleration rate is assumed to fall down below $0.5 \mathrm{~ms}^{-2}$ for speeds between $30 \mathrm{~km} / \mathrm{h}$ and the speed limit (usually $50 \mathrm{~km} / \mathrm{h}$ ). Running conditions are thus considered as cruising.

\subsection{Sound propagation model}

The proposed approach can handle with any sound propagation model depending on the urban landscape. In this paper, multiple reflections and diffractions are neglected since the studied roundabout is in open area. The sources associated with each vehicle are mapped on a set of moving line sources whose angle $\Theta_{n}^{t}$ is defined by the positions of vehicle $n$ at the beginning and the end of the time-step. Although this noise source representation requires an attenuation calculation at each time-step, it was selected because of its accuracy. Note that other classical source representations such as point source or fixed line source could have also been chosen [26]. Subsequently, the instantaneous noise pressure level $L_{A e q, \Delta t}^{t}$ at a receptor point $P$ is given by:
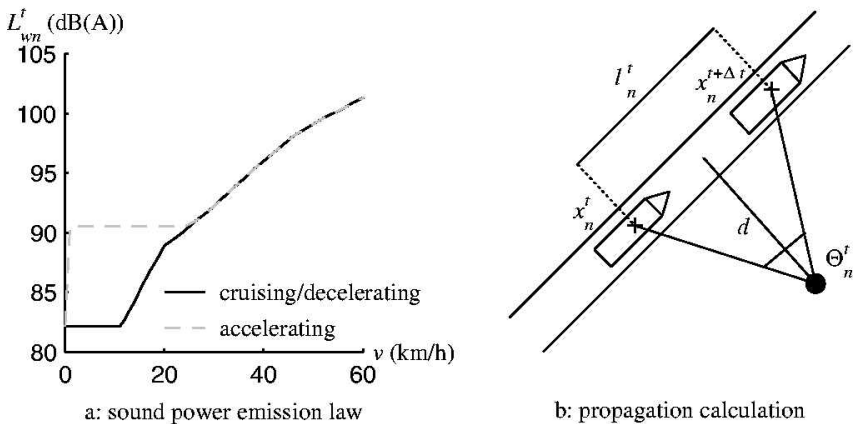

Fig. 4. Sound power levels and source representation. 
$L_{\text {Aeq, }, \Delta t}^{t}=10 \log \left(\sum_{n} \frac{\Theta_{n}^{t}}{2 \pi d\left\|t_{n}^{t}\right\|} 10^{\frac{L_{w n}^{t}}{10}}\right)$

where $\left\|I_{n}^{t}\right\|$ is the length of the line source; $d$ is the distance between $P$ and the line source (see Fig. $4 \mathrm{~b}$ ).

\section{Noise emission model validation}

\subsection{Study site}

To make a validation of the proposed dynamic noise emission model, a single-lane roundabout in the suburban area of Toulouse, France, was chosen as a study area. The goal is to check the relevance of the simulated dynamic noise contour maps by comparing observed and simulated sound pressure levels at several receivers. The emissions comparison is focused on one quarter of the roundabout with one receiver along the approach 4 , one receiver between the approach 4 and the next departure link 1 and 2 receivers along the departure link 1 (see Fig. 5). Due to symmetry effects, it is assumed that if the simulated dynamic noise levels are accurate at these receivers, so they are along the other legs of the roundabout.

\subsection{Data collection process}

The studied site is a four-leg roundabout in an open area without high buildings in the surroundings as shown in Fig. 5a. Traffic flow is moderate and essentially composed of light duty vehicles. Buses and heavy duty vehicles represent only $1.9 \%$ (respectively, $0.9 \%$ ) of the overall traffic in the morning (respectively in the evening) while motorcycles represent $1.3 \%$. These figures justify the use of a single mean emission law to characterize all vehicles on the roundabout. The main movements are from entry 1 to exit 3 in the morning and from entry 3 to exit 1 in the evening. Note that receivers 1 and 2 were placed to measure how noise levels are affected by the disturbance effect of the main movement (1-3) on the stream coming from approach 4.

Two sets of traffic and acoustical data were collected on $2 \mathrm{~h}$ periods in the morning and in the evening peaks in October 2005. Traffic data was collected from a $15 \mathrm{~m}$-high video camera which has recorded movements of all vehicles. An image processing software (called AUTOSCOPE) was used to extract passing times and vehicle identities at about $4 \mathrm{~m}$ before each entry $\left(A_{1-}\right.$ $A_{4}$ ) and after each exit $\left(D_{1}-D_{4}\right)$ (see Fig. 5b). Two additional positions were analyzed on link 1 at, respectively 30 and $60 \mathrm{~m}$ from the yield line $\left(A_{30}, A_{60}, D_{30}, D_{60}\right)$. In the microscopic traffic simulation tool, incoming traffic on each approaching link is reproduced from the passing times at $A_{60}, A_{2}, A_{3}$ and $A_{4}$. Moreover, each created vehicle is assigned a destination thanks to the vehicle identities tracking at each entry and exit. The four microphones were located in the acoustic field at $1.2 \mathrm{~m}$ high and at $5-6.5 \mathrm{~m}$ from the road axis (see Fig. 5b). The sound pressure levels were recorded every one $\operatorname{second}(\Delta t=1 \mathrm{~s})$ in global and in third-octave band spectra over the range $63-10,000 \mathrm{~Hz}$.

\subsection{Parameters calibration}

Table 2 summarizes the parameter values chosen for the microscopic traffic flow simulation model. They were fitted for matching observed traffic data or falling in the range of classical recommended values for urban conditions.

The free-flow speed $u$ on the approaching and departure arms has been set equal to the speed limit with an extra of $5 \%$ (to account for non-complying vehicles). Inside the roundabout, it has been chosen according to the recorded vehicle passing times when traffic volume is low; note that it matches classical recommended values for single lane roundabouts usually comprised between $20 \mathrm{~km} / \mathrm{h}$ and $24 \mathrm{~km} / \mathrm{h}$ [27]. The desired acceleration rate $a$ has been calibrated from observed vehicle trajectories on the departure arm 1. Parameter $w$ is characteristic of a one-way urban link and can be measured by estimating the starting wave speed at a signalized intersection when signal turns green, for instance by video-recording the location the back of the queue in time [28]. Parameter $s_{0}$ is also characteristic of a one-way urban link and can be determined from the average spacing between two stopped vehicles. An average value of $2 \mathrm{~km} / \mathrm{h}$ for the parameter $\epsilon$ was found to provide accurate vehicle trajectories in Leclercq et al. [29] and has been chosen in this study since the relaxation phenomenon seems to be nonsite-specific.

Parameters $t_{f}$ and $t_{c}$ were chosen so as to be in agreement with the recommended values of the American National Cooperative Highway Research Program [30] based on extensive validation studies on single-lane roundabouts:

Table 2

Calibration of the traffic flow simulation tool

\begin{tabular}{llllll}
\hline & & Units & $\begin{array}{l}\text { Approach } \\
\text { links }\end{array}$ & $\begin{array}{l}\text { Departure } \\
\text { links }\end{array}$ & $\begin{array}{l}\text { Circulatory } \\
\text { roadway }\end{array}$ \\
\hline Car-following & $u$ & $(\mathrm{~km} / \mathrm{h})$ & 44.6 & 54 & 19 \\
& $s_{0}$ & $(\mathrm{~m})$ & & 4.8 & \\
& $w$ & $(\mathrm{~km} / \mathrm{h})$ & & -10.6 & \\
Insertion decision & $\epsilon$ & $(\mathrm{km} / \mathrm{h})$ & 2 & \\
& $a$ & $\left(\mathrm{~m} / \mathrm{s}^{2}\right)$ & & 1.3 & \\
& $t_{f}$ & $(\mathrm{~s})$ & & 3 & \\
& $t_{c}\left(d_{\text {lag }}\right)$ & $(\mathrm{s})(\mathrm{m})$ & & $5(10.6)$ & \\
& $\gamma$ & $\mathrm{No}$ & & 1 & \\
\hline
\end{tabular}

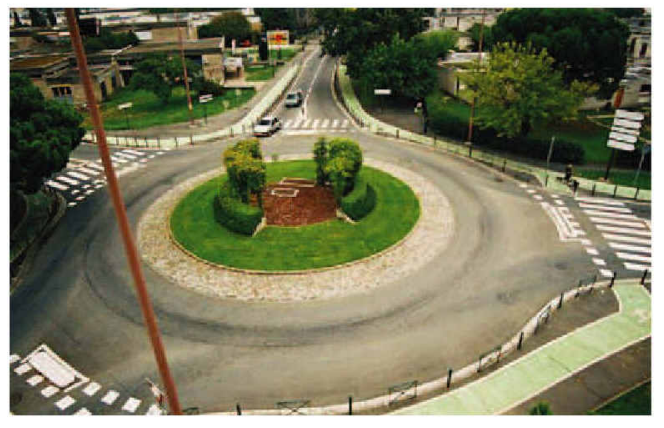

a: studied roundabout

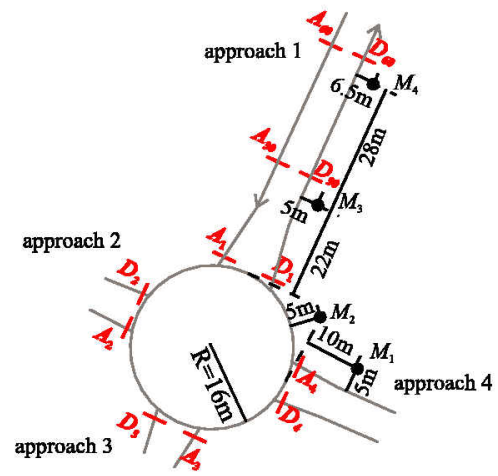

b: traffic and acoustic data collection points

Fig. 5. Data collection process. 
- $t_{f}$ is usually comprised between $2.6 \mathrm{~s}$ and $4.3 \mathrm{~s}$ with an average of $3.2 \mathrm{~s}$ and a standard deviation of $1.1 \mathrm{~s}$;

- $t_{c}$ is usually comprised between $4.2 \mathrm{~s}$ and $5.9 \mathrm{~s}$ with an average of $5.1 \mathrm{~s}$ and a standard deviation of $1.3 \mathrm{~s}$.

Finally, parameter $\gamma$ has been set equal to 1 since, when the roundabout is congested vehicles have been observed to share priority with circulating vehicles given a one-by-one process.

\subsection{Noise descriptors selection for the validation study}

Prediction of the instantaneous noise pressure levels $L_{\text {Aeq, 1s }}^{t}$ offers a substantial breakthrough compared to analytical estimation models. Firstly, the energy-based descriptors can be calculated eas- ily over any aggregation period. In the sequel, the equivalent sound pressure level, $L_{\text {Aeq }}$, will be computed over the entire $2 \mathrm{~h}$-simulation periods and over successive 15 min-intervals. Secondly, statistical descriptors, $L_{N T}$, reflecting the A-weighted pressure level exceeded during $N \%$ of the aggregation period $T$, can be evaluated. We will study the $L_{5, T}, L_{10, T}, L_{50, T}$ and $L_{90, T}$ on $2 \mathrm{~h}$ and 15 min-periods. Note that shortest aggregation periods were shown to be unreliable in Can et al. [31] because of the descriptors' sensitivity to noise peaks and the lack of statistical significance of the $L_{A e q}$ sample. Thirdly, the distribution of the $L_{\text {Aeq, } 1 \text { s }}^{t}$ over the entire simulation period can be obtained. This is a valuable tool for assessing the ability of the proposed model to reproduce dynamic on-field noise measurement. Finally, the time-series of $L_{\text {Aeq.1s }}^{t}$ can be used to predict when the noisiest periods will occur. Particularly, one

Table 3

Energy-based and statistical descriptors on $2 \mathrm{~h}$-periods

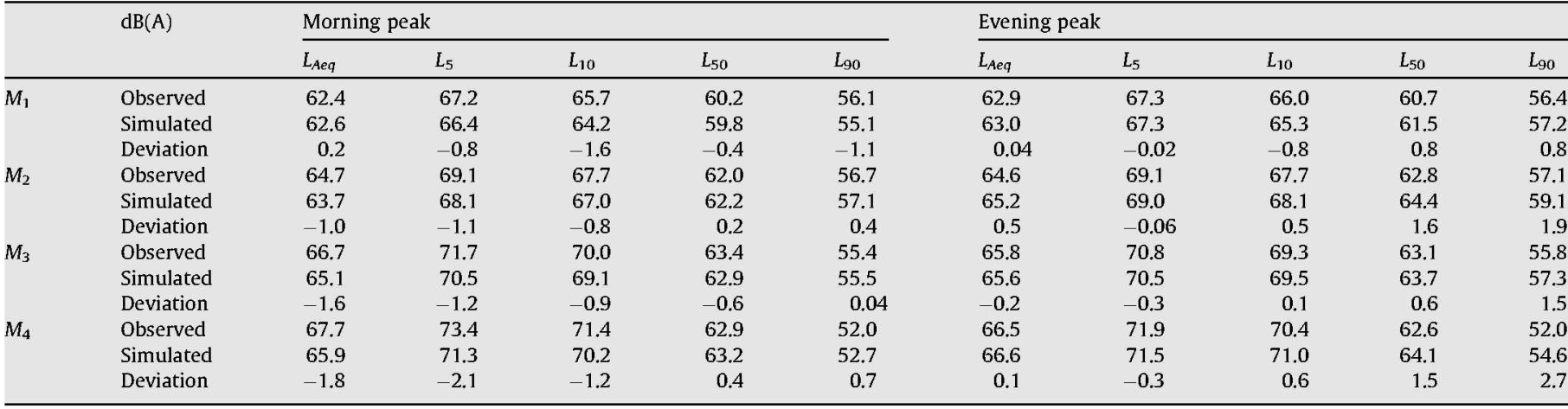
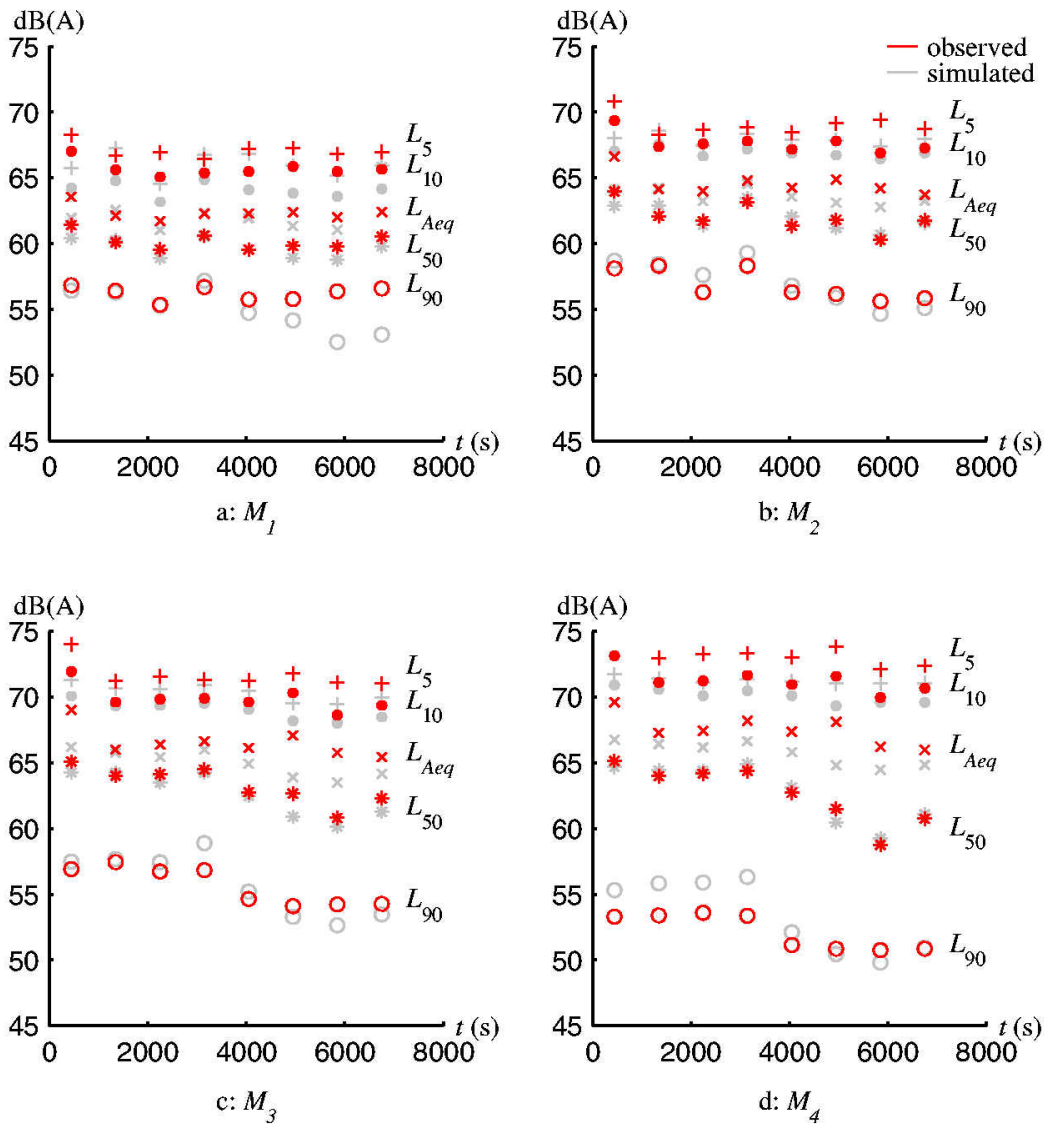

Fig. 6. Time-evolution of noise descriptors over 15 min-periods in the morning peak. 
is able to pinpoint which traffic situations have the greatest impacts in noise levels. This can help in the choice of traffic management policies or infrastructure design.

\subsection{Results}

\subsubsection{Energy-based and statistical descriptors on $2 \mathrm{~h}$-periods}

Table 3 sums-up the equivalent sound pressure level and the classical statistical descriptors aggregated over each $2 \mathrm{~h}$-simulation period, both for measurements and simulations. In all 40 cases but two, descriptors are estimated within a $2 \mathrm{~dB}(\mathrm{~A})$ error bound. The maximum deviation is $2.7 \mathrm{~dB}(\mathrm{~A})$. More specially, the deviation in $L_{\text {Aeq }}$ never exceeds $1 \mathrm{~dB}(\mathrm{~A})$ for all microphones except $M_{3}$ and $M_{4}$ in the morning peak where it reaches a $1.8 \mathrm{~dB}(\mathrm{~A})$ underestimation. A similar accuracy is observed for $L_{50}$. Higher noise levels corresponding to $L_{5}$ and $L_{10}$ are also reproduced with a precision of $1 \mathrm{~dB}(\mathrm{~A})$ for all microphones in the evening peak. The discrepancy is slightly higher in the morning peak with an underestimation lying from $0.8 \mathrm{~dB}(\mathrm{~A})$ to $2.1 \mathrm{~dB}(\mathrm{~A})$. Lowest noise levels characterized by $L_{90}$ fall within a $1 \mathrm{~dB}(\mathrm{~A})$ error bound in the morning peak. They are slightly overestimated from $0.8 \mathrm{~dB}(\mathrm{~A})$ to $2.7 \mathrm{~dB}(\mathrm{~A})$ in the evening peak.

\subsubsection{Time-evolution of energy-based and statistical descriptors on 15 min-periods}

The previous statistical descriptors are now aggregated over 15 min-periods and their time-evolution is compared in Figs. 6 and 7.

Since flows are roughly constant during each $2 \mathrm{~h}$-peak period, statistical levels are not varying a lot from one aggregation period to the other. One can notice that the simulated results closely match the time-evolution of the observed levels. Particularly, the maximum deviation for all descriptors except $L_{90}$ never exceeds $3.5 \mathrm{~dB}(\mathrm{~A})$ in the morning peak and $2.7 \mathrm{~dB}(\mathrm{~A})$ in the evening peak. Most of the estimates usually fall within a $1 \mathrm{~dB}(\mathrm{~A})$ accuracy compared to measurement. Although trucks and buses are disregarded, the $L_{5}$ level is correctly estimated by the model, which confirms that such noisy vehicles were nearly absent from the study site. In other circumstances, they should be included to improve prediction of the highest noise levels. This could be achieved with the proposed dynamic noise prediction tool since: (i) the traffic flow model can be extended to represent the effects of heavy vehicles on traffic [32]; (ii) specific French noise emission laws for heavy vehicles have been derived [33]. It should be worth also to notice that the model seems to be less effective in predicting the $L_{90}$ time-evolution on 15 min-intervals even if the discrepancy never exceeds $4 \mathrm{~dB}(\mathrm{~A})$. This is not surprising since the model only accounts for road traffic noise and neglects disturbances coming from other sources such as wind, pedestrians or cyclists (see Fig. 7).

\subsubsection{Distributions of the instantaneous sound pressure levels}

Figs. 8 and 9 show the distribution of the instantaneous sound pressure levels over both $2 \mathrm{~h}$-simulation periods at the four receptor points. Whatever the receiver, the shape of the simulated distribution is very close to the observed one. Particularly, the most frequent simulated levels perfectly match the most frequent observed modes. For the microphones $M_{3}$ and $M_{4}$ mostly affected by accelerating vehicles on the main departure link, one can see that the noisiest levels are usually under-represented by the model. Two reasons can explain this phenomena: (i) heavy vehicles and motorcycles which are expected to be noisier than light-duty vehicles, especially in accelerating mode, are not accounted for in this
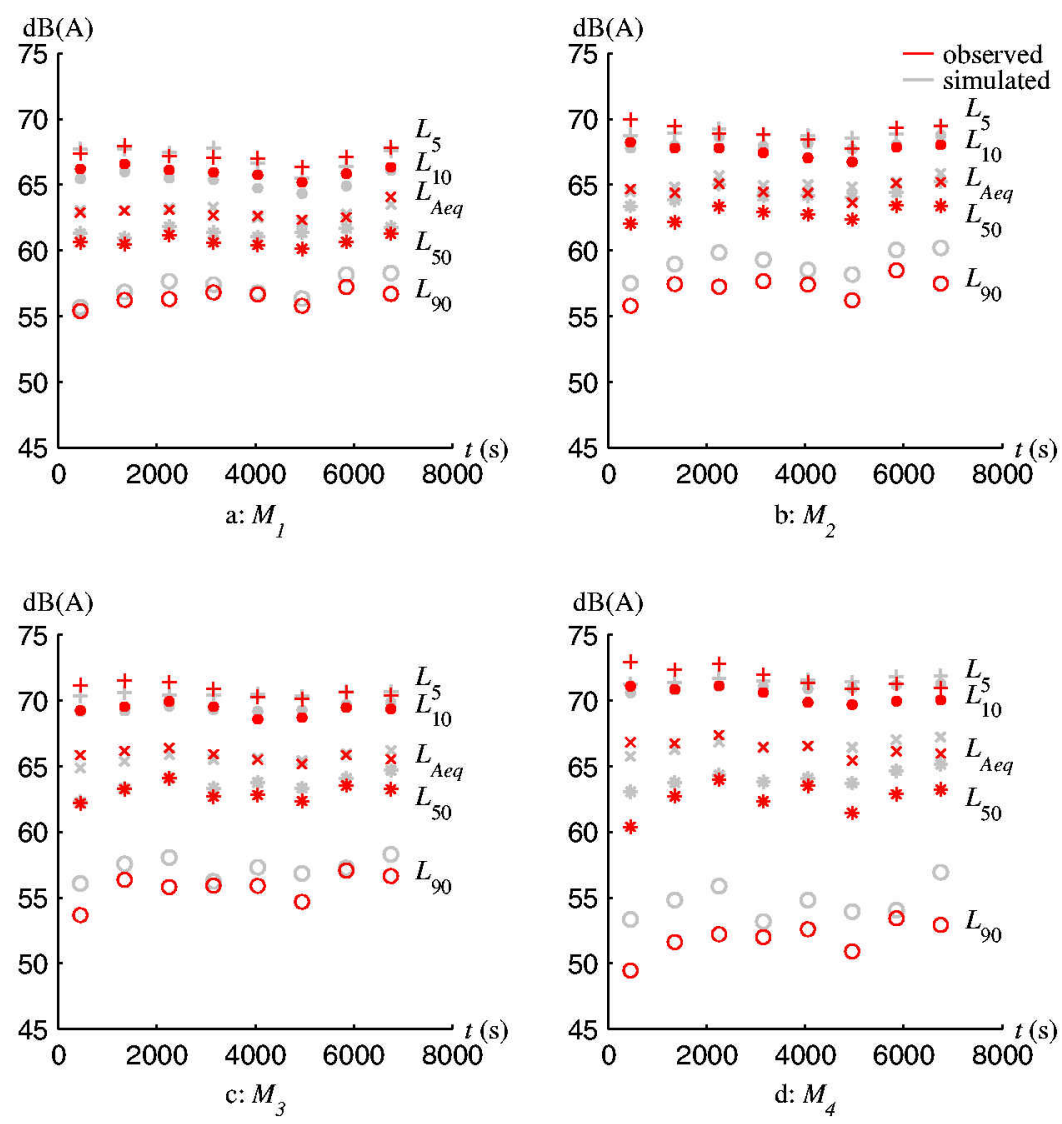

Fig. 7. Time-evolution of noise descriptors over 15 min-periods in the evening peak. 

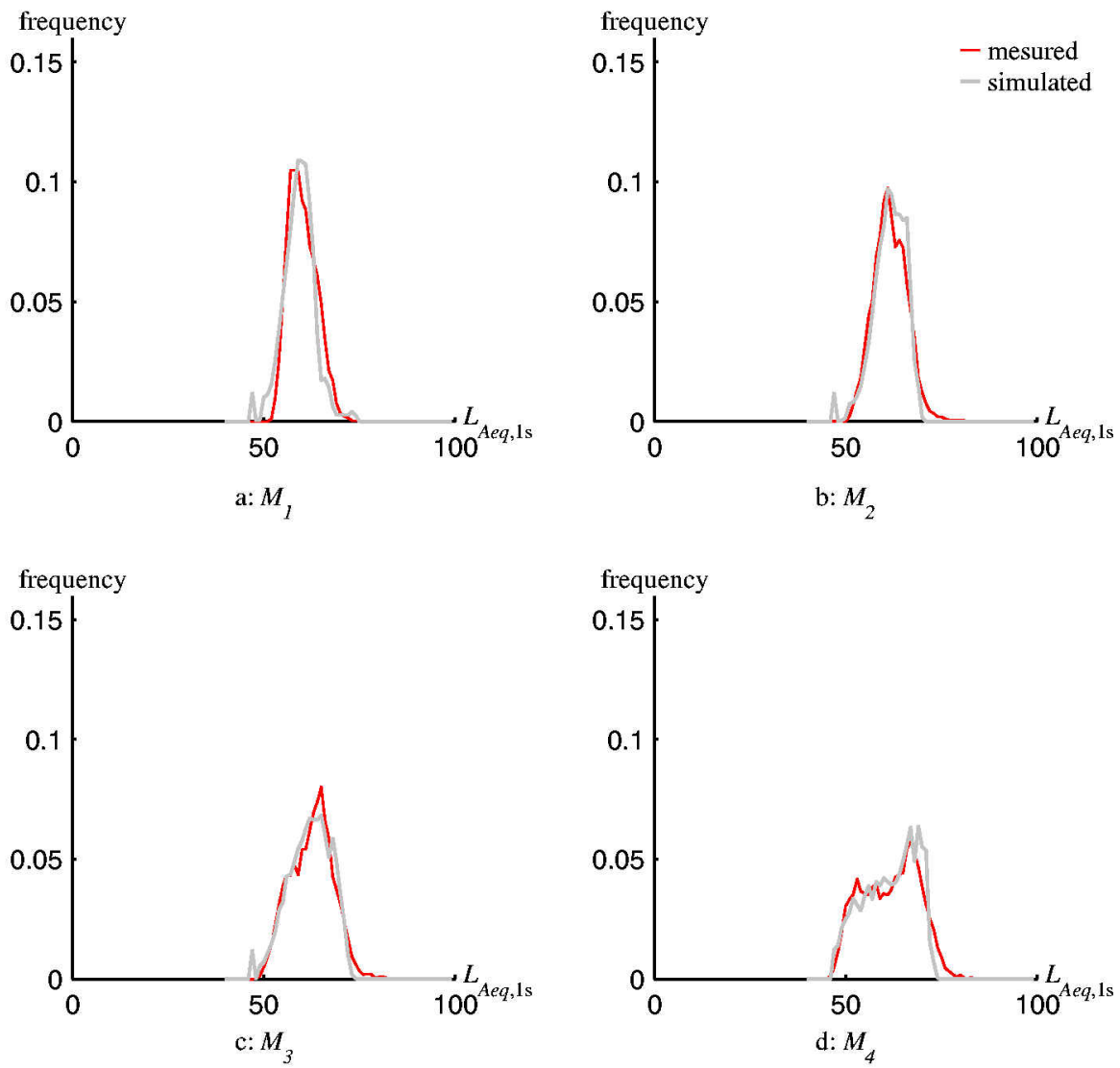

Fig. 8. Statistical distribution of the instantaneous sound pressure levels in the morning peak.
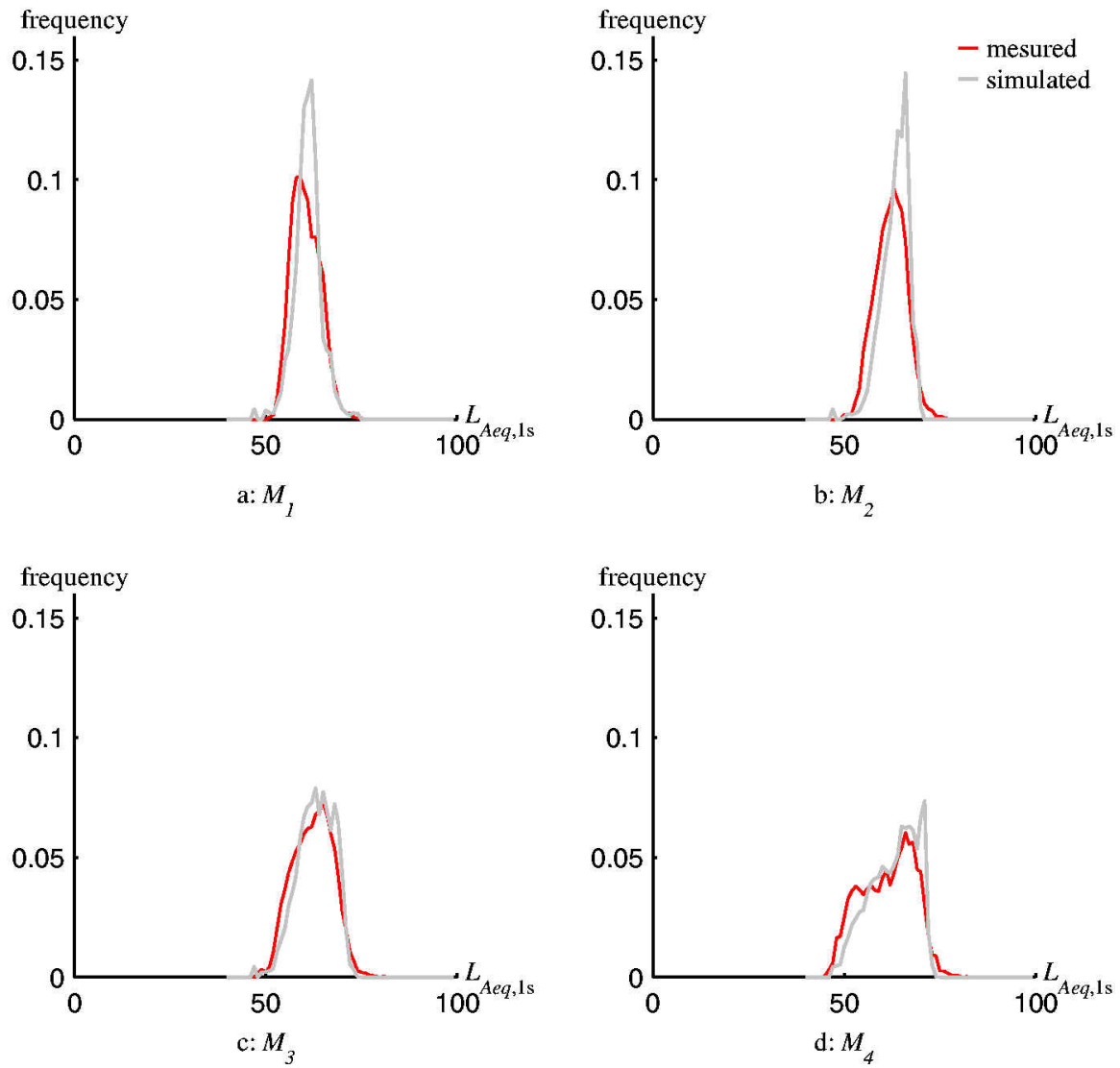

Fig. 9. Statistical distribution of the instantaneous sound pressure levels in the evening peak. 
study; (ii) random events like klaxons, road works or human voices in front of a microphone are disregarded. Moreover, for all receivers, the lowest levels are slightly over-represented. Indeed, between the passing by of vehicles, the simulated level drops to the background noise level. This explains the little peaks in the simulated distribution for levels around $47 \mathrm{~dB}(\mathrm{~A})$. In reality, low levels are more scattered due to noise coming from other sources (see Fig. 9).

\subsubsection{Noise contour maps}

Building a rectangular grid of receivers with spacing of $4 \mathrm{~m}$ allows us to generate dynamic noise contour maps of sound pressure levels at each time-step. They can be used to draw the time-evolution of noise contour maps of the $L_{A e q}$ and all statistical descriptors over a given sub-period. Maps of the $L_{\text {Aeq }}$ and $L_{10}$ over the first 15 min-period of the morning peak period are illustrated in Fig. 10. One can see that the highest levels of both $L_{A e q}$ and $L_{10} \mathrm{OC}-$ curs on the main link 1 , at the exits and entries. They reach values of $65 \mathrm{~dB}(\mathrm{~A})$ for the $L_{A e q}$ and of $70 \mathrm{~dB}(\mathrm{~A})$ for the $L_{10}$ at $5 \mathrm{~m}$ from the road axis of link 1 . Those values decrease on the circulating road- way and farther from link 1 . Note that noise levels around link 4 are much lower because of fewer arriving and departing vehicles on this link.

Analysis of dynamic noise contour maps can also be valuable to identify the nosiest traffic situations. For instance, two situations, with about the same number of vehicles on the roundabout, are depicted in Fig. 11. On the left side, only one vehicle is approaching fast on link 4 since it is not disturbed by circulating vehicles. Consequently, the noise levels received close to link 4 are important. On the right side of the figure, three vehicles are queuing because of hindrance of circulating vehicles. Due to their very low speeds, the noise levels are lower than previously even if more vehicles are present on the roundabout. This example shows that vehicle interactions have a substantial effect on noise levels. Analytical methods which neglect them are therefore inconsistent.

\section{Conclusion}

The proposed dynamic noise emission model fills the shortage of accurate noise estimation procedures at roundabouts. The noise

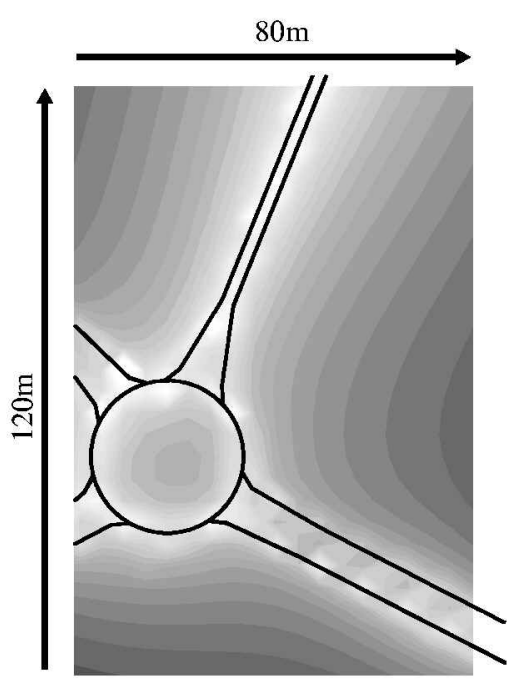

a: $L_{\text {Aeg, Ismn }}$ in the morning

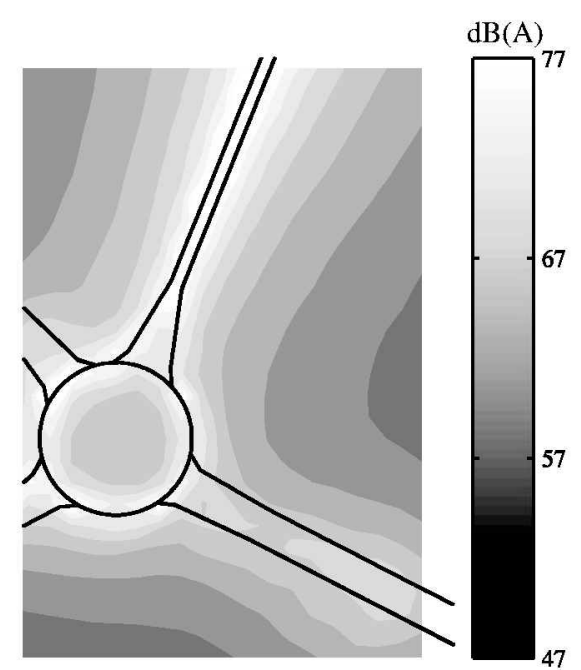

b: $L_{10,15 \mathrm{mn}}$ in the morning

Fig. 10. Noise contour maps of descriptors over the first 15 min-period in the morning peak.

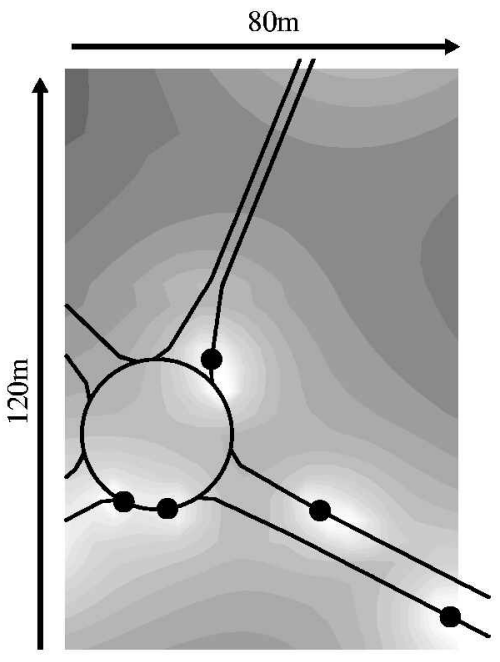

a: free-running vehicles on link 4
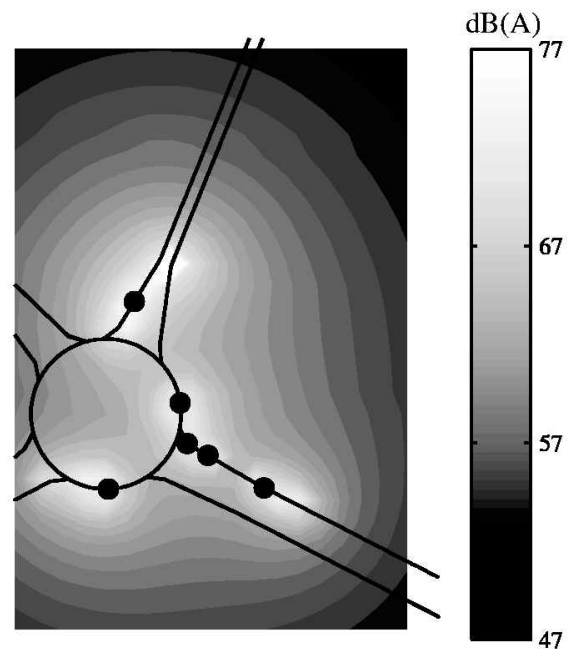

b: queuing vehicles on link 4

Fig. 11. Dynamic noise contour maps of events occurring in the evening peak 
emissions due to stochastic vehicle interactions at roundabout entries can be fully captured by combining a microscopic traffic flow model with noise emission laws and propagation calculation. Contrary to other existing noise simulation packages, the merging process and its impact on kinematics, vehicle delays and queue lengths is accurately reproduced, whatever the traffic conditions. The traffic outputs are fed into relevant noise emission laws depending on the vehicle type and the prevailing road pavement type. From vehicle speed and acceleration an instantaneous noise power level can be calculated for each vehicle. These levels are input into a propagation model to compute the noise levels received at different points in the vicinity of the roundabout. Accuracy of the obtained noise contour maps was demonstrated by comparing the simulated and the observed noise pressure levels at several receivers along a suburban roundabout. Such maps could be used to draw noise difference contours where the effects of the junction are compared with noise levels if vehicles were freely moving. This could help in deriving correction factors for simpler analytical noise prediction models to adjust for the extra noise induced by the junction. Moreover, noise difference contours could also be plotted for different junction layouts or control types to help practitioners for choosing the best design for the road-crossing.

The roundabout noise emission model will be integrated into a more general noise simulation package of a whole network called SYMUBRUIT [34]. It will allow an explicit modeling of roundabouts while, up to now, SYMUBRUIT just treats junctions as pointwise intersections. Extension of the roundabout model to other types of unsignalized intersections as well as to non-priority turning movements at traffic signals would be worth investigating. It will complete the detailed modeling of intersections within SYMUBRUIT. Further validation studies should also be conducted to check the accuracy of estimated noise levels when several local streets, urban arterials and intersections interact together. This will open the door to better noise impacts assessment of local transport management policies such as junction control device, intersection layout or signal coordination plans.

\section{Acknowledgements}

The Toulouse experimental study was funded by the Centre for the Study of Urban Planning, Transport and Public Facilities (CERTU) and managed by the ZELT laboratory. The authors are also grateful to Arnaud Can for his advice in the acoustic data processing as well as to Cécile Bécarie for coding the model. Finally, many thanks to the two anonymous referees for helping to greatly improve this article.

\section{References}

[1] IMAGINE. Review of the suitability of traffic models for noise modelling WP2: demand and traffic flow modelling. Project No. 503549 funded by the CE under the sixth framework programme; 2004. p. 132.

[2] Steele C. A critical review of some traffic noise prediction models. Appl Acoust 2001;62(3):271-87.

[3] Fleming GG, Rapoza AS, Lee CSY. Development of national reference energy mean emission levels for the FHWA traffic noise model, version 1.0 . Publication No. DOT-VNTSC-FHWA-96-2, Office of Engineering and Highway Operations Research and Development; 1995. p. 452.

[4] RLS. Richtlinien für den Lärmschutz an Strassen. BM für Verkehr, Bonn, Germany: 1990.

[5] Abo-Qudais S, Alhiary A. Statistical models for traffic noise at signalized intersections. Build Environ 2007;42(8):2939-48.
[6] Hunt M, Samuels S. Prediction of traffic noise at signalized intersections: the Australian experience. In: International congress and exposition on noise control engineering (INTERNOISE), Toronto, Canada; 1992.

[7] Certu. French guide for transportation noise (Guide du bruit des transports terrestres - Prévision des niveaux sonores). CETUR, ISBN: 2-11-083290-8; 1980. p. 317.

[8] Picaut J, Bérengier M, Rousseau E. Noise impact modelling of a roundabout. In: International congress and exposition on noise control engineering (INTERNOISE), Rio de Janeiro, Brazil; 2005.

[9] Watts G. Harmonoise prediction model for road traffic noise. TRL Report PPR 034, Department for Transport Roads Policy Division, England; 2005. p. 81.

[10] IMAGINE. The noise emission model for European road traffic. Project No 503549 funded by the CE under the sixth framework programme; 2007. p. 66 .

[11] Bhaskar A, Chung E, Kuwahara M. Development and implementation of the areawide Dynamic ROad traffic NoisE (DRONE) simulator. Transport Res Part D 2007;12(5):371-8.

[12] De Coensel B, De Muer T, Yperman I, Botteldooren D. The influence of traffic flow dynamics on urban soundscapes. Appl Acoust 2005;66(2):175-94.

[13] Goodman PS. The prediction of road traffic noise in urban areas. PhD thesis, Institute for Transport Studies, University of Leeds, UK; 2001.

[14] Volkmar H. Development of a microscopic road traffic noise model for the assessment of noise reduction measures (ROTRANOMO). In: Final conference, Berlin, Germany, <www.rotranomo.com>; 2005.

[15] Hidas P. Lane changing and merging under congested conditions in traffic simulation models. In: Brebbia CA, Wadhwa LC, editors, Proceedings of urban transport XI - urban transport and the environment in the 21th century; 2005. p. 779-88.

[16] Chevallier E, Leclercq L. Macroscopic investigation of microscopic merging models at unsignalized intersections. In: Transportation research board, Washington, DC, USA; 2008.

[17] Smith MC, Sadek AW, Johnson I, Aultman-Hall L, Garder P. Assessing accuracy of roundabouts analysis procedures against field observation: preliminary results from a case study in Northern New England. In: Transportation Research Board, Washington, DC, USA; 2007.

[18] Akçelik R, Besley M. Microsimulation and analytical methods for modelling urban traffic. In: Conference on advance modeling techniques and quality of service in highway capacity analysis, Truckee, California, USA; 2001.

[19] Kyte M, Tian Z, Mir Z, Hameedmansoor Z, Kittelson W, Vandehey M, et al. Capacity and level of service at unsignalized intersections, final report: volume 1-two-way-stop-controlled intersections. In: National cooperative highway research program 3-46, Transportation Research Board, Washington, DC, USA; 1996.

[20] Cassidy MJ, Ahn S. Driver turn-taking behaviour in congested freeway merges. Transport Res Rec 2005;1934:140-7.

[21] Troutbeck RJ. The performance of uncontrolled merges using a limited priority process. In: Taylor M, editor. Proceedings of the 15th ISTTT; 2002. p. 463-82

[22] Laval J, Leclercq L. Microscopic modeling of the relaxation phenomenon using a macroscopic lane-changing model. Transport Res Part B 2008;42(6):511-22.

[23] Can A, Leclercq L, Lelong J. Dynamic urban traffic noise: do individualized emission laws improve estimation? In: 19th international congress on acoustics (ICA), Madrid, Spain; 2007.

[24] Hamet JF, Besnard F, Doisy S, Lelong J. New vehicle noise emission for French traffic noise prediction. Appl Acoust, in press.

[25] Leclercq L, Can A, Crepeaux P, Defrance J, Fournier M, Lelong J, et al. Estimation dynamique du bruit de circulation en milieu urbain: étude d'un cas réel. Rapport final de la convention ADEME No. 0504C006317. Bron: INRETS, Rapport INRETS/LICIT No. 0801; 2008. p. 224.

[26] Can A, Leclercq L, Lelong J. Dynamic estimation of urban traffic noise: influence of traffic and noise source representations. Appl Acoust 2008;69(10):858-67.

[27] Robinson BW. Roundabouts: an informational guide. Publication No. FHWARD-00-067, <http://www.tfhrc.gov>; 2000. p. 284.

[28] Leclercq L. Calibration of flow-density relationships on urban streets. Transport Res Rec 2005;1934:226-34.

[29] Leclercq L, Chiabaut N, Laval J, Buisson C. Relaxation phenomenon after changing lanes: an experimental validation with the NGSIM dataset. Transport Res Rec 2007;1999:226-34.

[30] Rodegerdts LA, Blogg M, Wemple E, Myers E, Kyte M, Dixon M, et al. Roundabout in the United States. Publication NCHRP Report 572; 2007. p. 125

[31] Can A, Leclercq L, Lelong J, Defrance J. Capturing urban traffic noise dynamics through relevant descriptors. Appl Acoust 2008;69(12):1270-80.

[32] Leclercq L, Laval J. A multiclass car-following rule based on the LWR model. In Traffic and granular flow, Paris, France; 2007. p. 735-53.

[33] Lelong J, Leclercq L. Dynamic evaluation of the noise emitted by a bimodal (passenger cars/buses) traffic flow. In: 32nd international congress and exposition on noise control engineering (INTERNOISE), Seogwipo, Korea; 1999.

[34] Leclercq L, Lelong J. Dynamic evaluation of urban traffic noise. In: Proceedings of the 17th international congress on acoustics, Rome, Italy; 2001. 